\title{
Kruskal Dynamics for Radial Geodesics
}

\author{
Abhas Mitra ${ }^{1,2}$ \\ ${ }^{1}$ Theoretical Astrophysics Section, Bhabha Atomic Research Centre, Mumbai, India \\ ${ }^{2}$ Homi Bhabha National Institute, Mumbai, India \\ Email: amitra@barc.gov.in
}

Received February 25, 2012; revised March 28, 2012; accepted April 24, 2012

\begin{abstract}
The total spacetime manifold for a Schwarzschild black hole (BH) is believed to be described by the Kruskal coordinates $u=u(r, t)$ and $v=v(r, t)$, where $r$ and $t$ are the conventional Schwarzschild radial and time coordinates respectively. The relationship between $r$ and $t$ for a test particle moving along a radial or non-radial geodesic is well known. Similarly, the expression for the vacuum Schwarzschild derivative for a geodesic, in terms of the constants of motion, is well known. However, the same is not true for the Kruskal coordinates; and, we derive here the expression for the Kruskal derivative for a radial geodesic in terms of the constants of motion. In particular, it is seen that the value of $|\mathrm{d} u / \mathrm{d} v|(=1)$ is regular on the Event Horizon of the Black Hole. The regular nature of the Kruskal derivative is in sharp contrast with the Schwarzschild derivative, $|\mathrm{d} t / \mathrm{d} r|=\infty$, at the Event Horizon. We also explicitly obtain the value of the Kruskal coordinates on the Event Horizon as a function of the constant of motion for a test particle on a radial geodesic. The physical implications of this result will be discussed elsewhere.
\end{abstract}

Keywords: Black Hole; Kruskal Coordinates; Spacetime Singularity

\section{Introduction}

It is known for more than 90 years that the region exterior to a point mass or the event horizon $\left(r>r_{g}=2 m\right)$ of a Schwarzschild Black Hole (BH) can be described by the vacuum Schwarzschild metric[1,2]:

$$
\mathrm{d} s^{2}=g_{t t} \mathrm{~d} t^{2}+g_{r r} \mathrm{~d} r^{2}+g_{\theta \theta} \mathrm{d} \theta^{2}+g_{\phi \phi} \mathrm{d} \phi^{2}
$$

where $g_{t t}=(1-2 m / r), g_{r r}=-(1-2 m / r)^{-1}, g_{\theta \theta}=-r^{2}$, and $g_{\phi \phi}=-r^{2} \sin ^{2} \theta \quad(G=c=1)$. Here, we are working with a spacetime signature of $+1,-1,-1,-1$ and $r$ has a distinct physical significance as the invariant area radius; i.e. $A=4 \pi r^{2}$ indicates invariant/scalar area of symmetric 2-surfaces. Clearly, then, by definition, the point mass having $A=0$ is at $r=0$, and hence, the scalar $r$ should continue to be a space-like coordinate even for $r \leq 2 \mathrm{~m}$. The coordinate time $t$ too has a physical significance as the proper time of a distant inertial observer $S_{\infty}$. At $r=2 m, g_{r r}$ blows up and as $r<2 m$, the $g_{t t}$ and $g_{r r}$ suddenly exchange their signatures and the metric acquires a signature $-1,+1,-1,-1$. Such an inversion of signature appears to be unphysical because (1) by, definition, $r$ is spacelike and (2) by principle of equivalance, there must be a local inertial frame where the metric is Lorenzian with the original signature $+1,-1,-1,-1$. Further though, the signature of $g_{r r}$ would change if there would be region beneath the Event
Horizon, $r=2 m$, the signatures of $g_{\theta \theta}$ and $g_{\phi \phi}$ remain unchanged. Despite such fundamental physical inconsistencies, in the black hole (BH) paradigm, it is believed that the $\mathrm{EH}$ is merely a coordinate singularity because the Kretschmann scalar is finite there. But it can easily be seen that, $K_{E H} \propto m^{-4}$ [3], and the idea that $K_{E H}$ is finite presumes that the integration constant appearing in the vacuum Schwarzschild solution $\alpha=2 \mathrm{~m}$, is finite. However, it has been shown that, though $\alpha=2 m>0$ is indeed finite for an object with radius $r_{b}>2 m$, such as the Sun or a neutron star, $\alpha=2 m=0$ for a neutral point particle with $r_{b}=0$ [4-7]. Yet, in order to progress, we ignore this scientific fact and continue to work in the artificial and incorrect mathematical paradigm of BHs.

The detail dynamics of a "test particle" in the vacuum external spacetime is well known for a very long time and discussion on it is contained in practically every text book or monograpgh on classical General Theory of Relativity (GTR) [1,2]. One of the key aspects for studying the kinematics of a test particle is the knowledge about the relevant derivative of the spatial coordinate with the temporal one. For instance for any geodesic having angular momentum or not, one knows the details about the behaviour of the Schwarzschild derivative $\mathrm{d} r / \mathrm{d} t$ or $\mathrm{d} t / \mathrm{d} r$. And the fact that $\mathrm{d} t / \mathrm{d} r=-\infty$ blows up at the 
Event Horizon restricts the utility of the Schwarzschild dynamics below the EH. This tantamounts to the well known fact that the vacuum Schwarzschild metric fails to describe the spacetime inside $r \leq 2 \mathrm{~m}$. Some authors, accordingly, swap the nomenclatures $r$ and $t$ inside the $\mathrm{EH}$. But this is inconsistent because the location of the (1) $\mathrm{EH}$ is still denoted by $r=2 m$ and not by $t=2 m$ and (2) by initial definition, the point mass resides at $r=0$ and not at $t=0$.

On the other hand, it has been claimed since 1960 that both the exterior and the interior regions of a $\mathrm{BH}$ may be described by a one-piece coordinate system suggested by Kruskal and Szekeres [8,9]. In this case too, the nomenclatures of $r$ and $t$ remain unchanged for $r<2 m$ unlike what is done by some authors in a desperate move to uphold the $\mathrm{BH}$ paradigm. Though, in the intervening 42 years hundreds of articles have been written on Kruskal coordinates, and most of the treatises on GTR too regularly deliberate upon the original work of Kruskal and Szekers, the fact remains that sufficient effort has not been made to study the kinematics of a test particle in terms of the Kruskal coordinates so that one could have a better insight and appreciation of the kinematics inside the EH. Accordingly, here, we would derive the expressions for the Kruskal derivative $\mathrm{d} u / \mathrm{d} v$ for a radial geodesic. For the sake of completeness, we shall start from the usual description about the Kruskal coordinates and first derive the exact expression for the value of the Kruskal coordinates on the $\mathrm{EH}\left(u_{H}\right.$ and $v_{H}$ ) in terms of $r$ and $t$. We shall show here that $u_{H}$ and $v_{H}$ are always non-zero and finite in general. More importantly, we shall explicitly show that unlike the Schwarzschild derivative, the Kruskal derivates are regular on the EH. Apparently this might be in accordance with the singularity free nature of the Kruskal coordinates. However, in a later paper, we shall show that this is, ironically, not the case.

\section{Kruskal Coordinates}

First note that, Kruskal coordinates are defined by presuming that the integration constant appearing in the vacuum Schwarzschild solution is non-zero in variance with the actual result that, even though for an object with $r>2 m$ (such as the Sun) $m>0$, for a point mass $m=0$ [3-7]. Yet, in this paper, we ignore this basic discrepency. For the region exterior to the EH (Sectors 1 \& $3)$, the Kruskal coordinates are defined as follows $[1,2]$ :

$$
\begin{aligned}
& u=f_{1}(r) \cosh \frac{t}{4 m} ; \\
& v=f_{1}(r) \sinh \frac{t}{4 m} ; r \geq 2 m
\end{aligned}
$$

where

$$
f_{1}(r)= \pm\left(\frac{r}{2 m}-1\right)^{1 / 2} e^{r / 4 m}
$$

Here the plus sign corresponds to "our universe" while the negative sign corresponds to the "other universe" [1, 2]. The "other universe" is a legitimate mathematical solution of the Schwarzschild problem irrespective of its observational reality (under the assumption $m>0$ ), and is a time reversed mirror image of "our universe".

And for the region interior to the horizon (Sectors $2 \&$ 4), we have

$$
\begin{aligned}
& u=f_{2}(r) \sinh \frac{t}{4 m} ; \\
& v=f_{2}(r) \cosh \frac{t}{4 m} ; r \leq 2 m
\end{aligned}
$$

where

$$
f_{2}(r)= \pm\left(1-\frac{r}{2 m}\right)^{1 / 2} e^{r / 4 m}
$$

In terms of $u$ and $v$, the metric for the entire spacetime is

$$
\begin{aligned}
\mathrm{d} s^{2}= & \frac{32 m^{3}}{r} e^{-r / 2 m}\left(\mathrm{~d} v^{2}-\mathrm{d} u^{2}\right) \\
& -r^{2}\left(\mathrm{~d} \theta^{2}+\mathrm{d} \phi^{2} \sin ^{2} \theta\right)
\end{aligned}
$$

The metric coefficients are regular everywhere (under the assumption $m>0$ ) except at the intrinsic singularity $r=0$. Since afterall the Kruskal coordinates are defined using $r$ and $t$, for a proper understanding of the Kruskal dynamics, it is necessary to recall the inter-relationship between the Kruskal and Schwarzschild coordinates for a radial geodesic.

\subsection{Inter Relation between Schwarzschild Coordinates}

For a test particle on a radial geodesic, the angular momentum is zero, and there is only one conserved quantity, the energy of the particle (per unit rest mass), E, as measured by a distant inertial observer:

$$
E \equiv \frac{\mathrm{d} t}{\mathrm{~d} s}(1-2 m / r)
$$

where $s$ is the proper time. For a massless particle like a photon, we have $E=\infty$, otherwise $E$ is finite. For a radial geodesic, the motion of the particle is determined by (see Chandrasekhar, p. 98) [10]

$$
\frac{\mathrm{d} r}{\mathrm{~d} s}=-\sqrt{E^{2}-(1-2 m / r)}
$$

and

$$
\frac{\mathrm{d} t}{\mathrm{~d} s}=\frac{E}{1-2 m / r}
$$


so that

$$
\frac{\mathrm{d} t}{\mathrm{~d} r}=-\frac{E(1-2 m / r)^{-1}}{\sqrt{E^{2}-(1-2 m / r)}}
$$

Clearly as $r \rightarrow 2 m, \mathrm{~d} t / \mathrm{d} r \rightarrow-\infty$. The latter relation $\mathrm{d} r / \mathrm{d} t=0$ may be indicating that the $r$ has a minimum at $2 m$. But since the minimum of $r$ is $r=0$, it may be indicating that, for a point mass $2 m=0 \quad$ [3-6]. However, we shall ignore this indication in this paper.

Here note that if the particle is released from rest $(\mathrm{d} r / \mathrm{d} s=0)$ at $r=r_{i}$ at $t=0$, from Equation (8), it is seen that $[10]$

$$
E^{2}=\left(1-2 m / r_{i}\right)
$$

or,

$$
r_{i} / 2 m=\left(1-E^{2}\right)^{-1}
$$

It is convenient to introduce a (cyclic) parameter $\eta$ through

$$
\begin{aligned}
r & =\frac{r_{i}}{2}(1+\cos \eta) \\
& =\frac{2 m}{1-E^{2}} \cos ^{2}(\eta / 2)=r_{i} \cos ^{2}(\eta / 2)
\end{aligned}
$$

Obviously, $\eta=0$ when $r=r_{i}$ and at the EH, we have

$$
\eta=\eta_{H}=2 \arcsin E ; \quad r=2 m
$$

Now after some manipulation, Chandrasekhar arrived at the following Equation involving $t$ and $\eta$ [10]:

$$
\frac{\mathrm{d} t}{\mathrm{~d} \eta}=E\left(\frac{r_{i}}{2 m}\right)^{1 / 2} \frac{\cos ^{4}(\eta / 2)}{\cos ^{2}(\eta / 2)-\cos ^{2}\left(\eta_{H} / 2\right)}
$$

This Equation can be integrated to find the exact relation between $t$ and $r$ for a radial geodesic (actually, even for non-radial geodesic this Equation would hold good):

$$
\begin{aligned}
& \frac{t}{2 m}=E\left(\frac{r_{i}}{2 m}\right)^{3 / 2}\left[\frac{1}{2}(\eta+\sin \eta)+\left(1-E^{2}\right) \eta\right] \\
& +\ln \left[\frac{\tan \left(\eta_{H} / 2\right)+\tan (\eta / 2)}{\tan \left(\eta_{H} / 2\right)-\tan (\eta / 2)}\right]
\end{aligned}
$$

The above Equation may also be written without introducing $\eta_{H}$ and $E$ explicitly: (see p. 824 of ref.[1] or p. 343 of ref. [2]):

$$
\begin{aligned}
& \frac{t}{2 m}=\ln \left|\frac{\left(r_{i} / 2 m-1\right)^{1 / 2}+\left(r_{i} / r-1\right)^{1 / 2}}{\left(r_{i} / 2 m-1\right)^{1 / 2}-\left(r_{i} / r-1\right)^{1 / 2}}\right| \\
& +\left(\frac{r_{i}}{2 m}-1\right)^{1 / 2}\left[\eta+\left(\frac{r_{i}}{4 m}\right)(\eta+\sin \eta)\right]
\end{aligned}
$$

We find from Equations (16) and (17) that, as $r \rightarrow 2 m$ from Sector 1, the logarithmic term blows up and $t \rightarrow \infty$, which is a well known result. Further Kruskal coordinates envisage that approach to the EH from the Sectors III \& IV corresponds to $t=-\infty$.

\subsection{Kruskal Coordinates on the Event Horizon}

In Sectors 1 \& 3, Kruskal coordinates obey the relation

$$
\frac{u}{v}=\operatorname{coth} \frac{t}{4 m}
$$

And since $r \rightarrow 2 m$ corresponds to $t \rightarrow \pm \infty$, at the $\mathrm{EH}$, we have

$$
\frac{u_{H}}{v_{H}}= \pm 1 ; \quad r=2 m
$$

On the other hand, in Sectors 2 and 4, we see

$$
\frac{u}{v}=\tanh \frac{t}{4 m}
$$

and as $r \rightarrow 2 m, t \rightarrow \pm \infty$, we are led to the same Equation (19). In the same limit, $r \rightarrow 2 m$ and $t \rightarrow \pm \infty$, we find that

$$
u_{H}^{2}=v_{H}^{2} \rightarrow f_{1}^{2} \exp \frac{t}{2 m}
$$

It might appear that since $f_{1}(2 m)=f_{2}(2 m)=0$ on the EH, we would have $u_{H}= \pm v_{H}=0$. But this is incorrect because the temporal part of $u$ and $v$ tends to blow up much more rapidly on the $\mathrm{EH}$. And one has to carefully obtain the actual values of $u_{H}$ and $v_{H}$ by working out appropriate limits

To do so we introduce a new variable

$$
z=r_{i} / 2 m-1=\frac{E^{2}}{1-E^{2}}
$$

and, let, in the vicinity of the EH,

$$
r / 2 m=1+\varepsilon ; \quad \varepsilon \rightarrow 0
$$

so that

$$
f_{i}^{2}(2 m) \rightarrow \varepsilon
$$

Then, in the vicinity of the $\mathrm{EH}$, by retaining terms first order in $\varepsilon$, we can rewrite Equation (17) as

$$
\begin{aligned}
& \frac{t}{2 m}=\ln \left|\frac{z^{1 / 2}+z^{1 / 2}\left(1-\frac{\varepsilon r_{i}}{4 m z}\right)}{z^{1 / 2}-z^{1 / 2}\left(1-\frac{\varepsilon r_{i}}{4 m z}\right)}\right| \\
& +\left(\frac{r_{i}}{2 m-1}\right)^{1 / 2}\left[\eta+\left(\frac{r_{i}}{4 m}\right)(\eta+\sin \eta)\right]
\end{aligned}
$$

As $\varepsilon \rightarrow 0$, the logarithmic term in the above expression becomes 


$$
A(t)=\ln \left|\frac{1+1-\frac{\varepsilon r_{i}}{4 m z}}{1-1+\frac{\varepsilon r_{i}}{4 m z}}\right| \rightarrow \ln \frac{8 m z}{r_{i} \varepsilon}
$$

Then, using Equations (24) and (26), we find

$$
f_{1}^{2} \exp (A) \rightarrow \frac{8 e m z}{r_{i}}=4 e\left(1-2 m / r_{i}\right)=4 e E^{2}
$$

Now considering the other terms in the expression for $t / 2 m$ in Equation (25), we find that, in this limit,

$$
\begin{aligned}
& u_{H}^{2}=v_{H}^{2}=4 e\left(1-2 m / r_{i}\right) \\
& \times \exp \left\{\left(\frac{r_{i}}{2 m}-1\right)^{1 / 2}\left[\eta_{H}+\left(\frac{r_{i}}{4 m}\right)\left(\eta_{H}+\sin \eta_{H}\right)\right]\right\}
\end{aligned}
$$

In terms of $E$, we have

$$
u_{H}^{2}=v_{H}^{2}=4 e E^{2} \exp E\left[\eta_{H}+\frac{\eta_{H}+\sin \eta_{H}}{2 \sqrt{1-E^{2}}}\right]
$$

One would have $u_{H}=v_{H}=0$ if $E=0$ or, if the test particle is injected from rest right at the EH. Clearly, this is unphysical, and thus we see that $u_{H}$ and $v_{H}$ are non-zero. Further, for a finite value of $r_{i} / 2 m$ or for $E<1$, they are finite too. The finiteness of $u$ and $v$ at the EH is physically appealing because $u$ and $v$ are expected to be completely regular at the EH. However for $r_{i} / 2 m=\infty$ or $E=1$, we find $u_{H}^{2}=v_{H}^{2}=\infty$.

On the other hand, since $r=r_{i}$ at $t=0$, by using the definition of $u$ and $v$, we find that the initial values of

$$
u^{2}=u_{i}^{2}=\left(r_{i} / 2 m-1\right)=\frac{E^{2}}{1-E^{2}}
$$

and

$$
v^{2}=v_{i}^{2}=0
$$

\section{Kruskal Derivative: A Direct Approach}

Having shown that $u_{H}$ and $v_{H}$ are, in general, nonzero, we are now in a position to evaluate the Kruskal derivative, the key ingredient for studying the Kruskal dynamics for a radial geodesic. We first confine ourselves to Sector 1. By differentiating $f_{1}(r)$ (Equation [3]) with $r$ we obtain

$$
\frac{\mathrm{d} f_{1}}{\mathrm{~d} r}=\frac{ \pm r}{2 m} \frac{e^{r / 4 m}}{4 m}(r / 2 m-1)^{-1 / 2}
$$

Then by directly differentiating Equation (2) by $r$, we find that irrespective of the sign of $\mathrm{d} f_{1} / \mathrm{d} r$, we will have

$$
\frac{\mathrm{d} u}{\mathrm{~d} r}=\frac{\mathrm{d} f_{1}}{\mathrm{~d} r} \cosh \frac{t}{4 m}+\frac{f_{1}}{4 m} \sinh \frac{t}{4 m} \frac{\mathrm{d} t}{\mathrm{~d} r}
$$

Interestingly, in all the sectors, we obtain the same functional form of $\mathrm{d} u / \mathrm{d} r$. Using Equations (2) and (4) in the foregoing Equation, we see that

$$
\frac{\mathrm{d} u}{\mathrm{~d} r}=\frac{u}{4 m}(1-2 m / r)^{-1}+\frac{v}{4 m} \frac{\mathrm{d} t}{\mathrm{~d} r}
$$

On the other hand by differentiating Equations (4) and (5), we find that

$$
\frac{\mathrm{d} f_{2}}{\mathrm{~d} r}=\frac{\mp r}{2 m} \frac{e^{r / 4 m}}{4 m}(r / 2 m-1)^{-1 / 2}
$$

and

$$
\frac{\mathrm{d} u}{\mathrm{~d} r}=\frac{\mathrm{d} f_{2}}{\mathrm{~d} r} \sinh \frac{t}{4 m}+\frac{f_{2}}{4 m} \cosh \frac{t}{4 m} \frac{\mathrm{d} t}{\mathrm{~d} r}
$$

And by using Equations (4) and (35) into the foregoing Equation, we obtain the same expression (34) for $\mathrm{d} u / \mathrm{d} r$ in Sectors 2 \& 4. Further, using Equation (10) in (34), we obtain the ultimate expression for

$$
\frac{\mathrm{d} u}{\mathrm{~d} r}=\frac{(1-2 m / r)^{-1}}{4 m}\left[u-\frac{v E}{\sqrt{E^{2}-1+2 m / r}}\right]
$$

valid in all the sectors. Similarly, we obtain the ultimate functional form of $\mathrm{d} v / \mathrm{d} r$ which is valid for all the sectors:

$$
\frac{\mathrm{d} v}{\mathrm{~d} r}=\frac{(1-2 m / r)^{-1}}{4 m}\left[v-\frac{u E}{\sqrt{E^{2}-1+2 m / r}}\right]
$$

And, the general value of $\mathrm{d} u / \mathrm{d} v$ in any Sector is obtained by dividing Equation (37) with (38):

$$
\frac{\mathrm{d} u}{\mathrm{~d} v}=\frac{u-\frac{v E}{\sqrt{E^{2}-1+2 m / r}}}{v-\frac{u E}{\sqrt{E^{2}-1+2 m / r}}}
$$

\subsection{Kruskal Derivative at the Event Horizon}

Since $u$ and $v$ are expected to be differentiable smooth continuous (singularity free) functions everywhere except at $r=0$ (under the assumption $m>0$ ), and also since the "other universe" is a mirror image of "our universe", we expect that the value of $\mathrm{d} u / \mathrm{d} v$ for any given $r$ must be the same, except for a probable difference in the signature, in both the universes. The meaningful way to find the value of $\mathrm{d} u / \mathrm{d} r$ at the EH will be to concentrate on the Sectors $2 \& 4$ for which $u_{H}=-v_{H}$

$$
\frac{\mathrm{d} u}{\mathrm{~d} v} \rightarrow \frac{u-v}{v-u}=\frac{2 u_{H}}{2 v_{H}}=-1 ; \quad r=2 m
$$

The Equation (39) for the Kruskal redivative, however, tends to yield a " $0 / 0$ " form at $r=2 m$ for Sectors $1 \& 3$ having $u_{H}=v_{H}$. But as mentioned above, we expect this $0 / 0$ form to acquire the value $\mathrm{d} u / \mathrm{d} v=+1$ because 
these Scetors are the mirror images of Sectors $2 \& 4$. Otherwise the whole idea of having an extended time symmetric Schwarzschild manifold would be inconsistent. Thus, in general, we must have

$$
\mathrm{d} u / \mathrm{d} v= \pm 1 ; \quad r=2 m
$$

despite the $0 / 0$ forms in some sectors. The fact that we must have $\mathrm{d} u / \mathrm{d} v=+1$ for the Sectors $1 \& 3$ can be reconfirmed in the limiting case of $u_{H}^{2}=v_{H}^{2}=\infty$ for $E=1$ or $u_{H}=v_{H}=0$ for the (unphysical case) $E=0$ directly by using L' Hospital's theorem.

Note that, by this rule, we can write,

$$
\lim _{u \rightarrow 0(\infty), v \rightarrow 0(\infty)} \frac{u}{v}=\lim _{u \rightarrow 0(\infty), v \rightarrow 0(\infty)} \frac{\mathrm{d} u / \mathrm{d} r}{\mathrm{~d} v / \mathrm{d} r}
$$

In any case, from Equation (19), we already know that $u / v= \pm 1$ at $r=2 m$. Then we can rearrange the foregoing Equation as

$$
\lim _{u \rightarrow 0(\infty), v \rightarrow 0(\infty)} \frac{\mathrm{d} u / \mathrm{d} r}{\mathrm{~d} v / \mathrm{d} r}= \pm 1
$$

or,

$$
\lim _{r \rightarrow 2 m} \frac{\mathrm{d} u}{\mathrm{~d} v}= \pm 1
$$

\section{A Different Route}

It may be of some interest to rederive the limiting value of $\mathrm{d} u / \mathrm{d} v$ by using other generic relationships between $u$ and $v$. As before, to avoid 0/0 forms, we work with Sectors $3 \&$ 4. In particular, in Sector 3, we have

$$
\frac{u}{v}=\operatorname{coth} \frac{t}{4 m}
$$

By differentiating this equation w.r.t. $v$, we obtain

$$
\frac{1}{v} \frac{\mathrm{d} u}{\mathrm{~d} v}-\frac{u}{v^{2}}=-\frac{1}{4 m} \frac{1}{\sinh ^{2}(t / 4 m)} \frac{\mathrm{d} t}{\mathrm{~d} v}
$$

By recalling that $\sinh (t / 4 m)=v / f_{1}$, we rewrite the above equation as

$$
\frac{\mathrm{d} u}{\mathrm{~d} v}-\frac{u}{v}=\frac{-f_{1}^{2}}{4 m} \frac{1}{v} \frac{\mathrm{d} t}{\mathrm{~d} v}
$$

Now, from Equations (10) and (38), note that

$$
\frac{\mathrm{d} t}{\mathrm{~d} v}=\frac{\mathrm{d} t}{\mathrm{~d} r} \frac{\mathrm{d} r}{\mathrm{~d} v}=-\frac{4 m E}{v \sqrt{E^{2}-1+2 m / r}-u E}
$$

And the limiting value of

$$
\begin{aligned}
& \frac{\mathrm{d} t}{\mathrm{~d} v} \rightarrow \frac{4 m}{u-v}=\frac{4 m}{u_{H}+u_{H}}=\frac{2 m}{u_{H}} \\
& r \rightarrow 2 m
\end{aligned}
$$

And since $f_{1}(2 m)=0$, we find from Equation (47) that

$$
\frac{\mathrm{d} u}{\mathrm{~d} v}-\frac{u}{v}=0 ; \quad r=2 m
$$

Or,

$$
\begin{aligned}
& \frac{\mathrm{d} u}{\mathrm{~d} v}=\frac{u}{v}=\frac{u_{H}}{-u_{H}}=-1 ; \\
& r=2 m(\text { Sectors } 3+4)
\end{aligned}
$$

Similarly, for the sake of overall consistency, in Sectors, $1 \& 3$, we must have $\mathrm{d} u / \mathrm{d} v=+1$ at $r=2 m$.

\section{A Different Consideration}

Actually we could have obtained the above derived unique result in a relatively simpler manner by differentiating the Global Equation

$$
u^{2}-v^{2}=(r / 2 m-1) e^{r / 2 m}
$$

w.r.t. $v$ :

$$
2 u \frac{\mathrm{d} u}{\mathrm{~d} v}-2 v=\frac{r}{4 m^{2}} e^{r / 2 m} \frac{\mathrm{d} r}{\mathrm{~d} v}
$$

First let us note from Equation (38) that in Sectors $2 \&$ 4 , the limiting value of

$$
\begin{aligned}
& \frac{\mathrm{d} r}{\mathrm{~d} v} \rightarrow \frac{4 m(1-2 m / r)}{v-u} \\
& \rightarrow \frac{2 m(1-2 m / r)}{v_{H}}=0 \\
& r \rightarrow 2 m
\end{aligned}
$$

Then, by using this above Equation in (53), we find

$$
u_{H} \frac{\mathrm{d} u}{\mathrm{~d} v}=v_{H} ; \quad r \rightarrow 2 m
$$

so that

$$
\frac{\mathrm{d} u}{\mathrm{~d} v}=\frac{v_{H}}{-v_{H}}=-1 ; \quad r \rightarrow 2 m
$$

\section{Conclusions}

The Kruskal coordinates were found way back in 1960 , and in the present paper, we have worked out some aspects of the kinematics of a test particle following a radial Kruskal geodesic. To attain this we used the precise value of $u_{H}$ and $v_{H}$ as a function of the initial conditions of the problem $r_{i}, m$ or $E$. It is clearly found that $u_{H}^{2}=v_{H}^{2}$ is non-zero in general.

We then proceeded to obtain the expression for the Kruskal derivative in terms of $m, E$ and $r$. We found that the Kruskal derivative is regular at the EH unlike the Scharzschild deivative(s) where $\mathrm{d} t / \mathrm{d} r=-\infty$ at the EH.

In particular $\mathrm{d} u / \mathrm{d} v=+1$ at the EH if we consider the 
"other universe" whose existence is suggested by the full Kruskal manifold, and which is a time reversed version of "our universe". But, if we move to the "our universe", the expected value of $\mathrm{d} u / \mathrm{d} v=-1$ at the EH.

The apparent regular nature of the Kruskal derivative may be seen to be in keeping with the notion that Kruskal coodinates are free of singularities at the EH. However, in a subsequent paper, we will delve into this question, and find out other important features of the Kruskal dynamics vis-a-vis the well known Schwarzschild dynamics.

Finally, this manuscript is a revision of the Cornell Univ. Electronic Preprint (arXiv: gr-qc/9909062) which has been viewed by many readers in the past 13 years.

\section{REFERENCES}

[1] C. W. Misner, K. S. Thorne and J. Wheeler, "Gravitation," Freeman, San Fransisco, 1973.

[2] S. L. Shapiro and S. A. Teukolsky, "Black Holes, White Dwarfs and Neutron Stars: The Physics of Compact Objects," Wiley, New York, 1983. doi:10.1002/9783527617661

[3] A. Mitra, "Black Holes or Eternally Collapsing Objects: A Review of 90 Years of Misconceptions," In: P. V.
Kreitler, Ed., Focus on Black Hole Research, Nova, New York, 2006.

[4] A. Mitra, "On the Non-Occurrence of Type I X-Ray Bursts from the Black Hole Candidates," Advances in Space Research, Vol. 38, No. 12, 2006, pp. 2917-2919. doi:10.1016/j.asr.2006.02.074

[5] A. Mitra, "Quantum Information Paradox: Real or Fictitious," Pramana, Vol. 73, No. 3, 2009, pp. 615-620. doi:10.1007/s12043-009-0113-9

[6] A. Mitra, "Comments on 'The Euclidean Gravitational Action as Black Hole Entropy, Singularities, and Space Time Voids'," Journal of Mathematical Physics, Vol. 50, No. 4, 2009, Article ID: 042501.

[7] A. Mitra, "The Fallacy of Oppenheimer Snyder Collapse: No General Relativistic Collapse at all, No Black Hole, No Physical Singularity," Astrophysics and Space Science, Vol. 332, No. 1, 2011, pp. 43-48. doi:10.1007/s10509-010-0578-5

[8] M. D. Kruskal, "Maximal Extension of Schwarzschild Metric," Physical Review Letters, Vol. 119, No. 5, 1960, pp. 1743-1745.

[9] P. Szekeres, "On the Singularities of a Riemannian Manifold," Math. Debreca., Vol. 7, 1960, p. 285.

[10] S. Chandrasekhar, "The Mathematical Theory of Black Holes," Clarendron, Oxford, 1983. 